\title{
Recent advances in computer modelling of granular systems
}

\author{
R. JULLIEN, P. MEAKIN* and A. PAVLOVITCH ${ }^{* *}$ \\ Laboratoire de Science des Matériaux Vitreux, Université Montpellier II, Place Eugène Bataillon, \\ 34095 Montpellier cedex 5, France \\ ${ }^{*}$ Physics Department, University of Oslo, P.O. Box 1048, Blindern, 0316 Oslo 3, Norway \\ ${ }^{* *}$ Section de Recherche de Métallurgie Physique, Centre d'Etudes de Saclay, BP. 2, 91191 Gif-sur-Yvette \\ cedex, France
}

\begin{abstract}
We present simple computer algorithms able to build random packings of spheres using the ballistic deposition model and we show how they can be used to investigate several size segregation phenomena occuring in granular systems: 1) penetration of a small sphere in a packing of large ones, 2) size-segregation in the formation of a heap or when pouring a silo, 3) size-segregation by shaking. In the last case, the computer simulation provides a very simple geometrical explanation of the phenomenon.
\end{abstract}

Random packings of spheres can be used as simple modelizations of granular materials. In many eases, the algorithms designed to generate random packings can be viewed as irreversible growth processes in which spheres (or discs in two dimensions) are added sequentially to the packings. Therefore the recent progresses made towards understanding of irreversible growth and aggregation phenomena[1] can provide new ideas on how to build random packings and to study some of their properties. Here, we report on some recent works based on a simple random deposition process, namely the ballistic model, which has been used to investigate several size segregation phenomena. The ballistic model, which was first introduced by Vold[2], was reinvented by Visscher and Bosterli[3] and later by two of $u s[4]$ in the context of aggregation and random deposition phenomena. Here we consider the fully restructured version of this model. In its monodisperse version, hard spheres (or discs in two dimension) of same diameter are released one after another, along randomly positionned vertical trajectories, onto a horizontal plane (or a line in two dimensions). As soon as the falling sphere touches the packing, it follows the path of steepest descent on its surface, rolling on spheres as often as necessary until it either touches the substrate or reaches a position in contact with three spheres which is in stable under gravity (or a stable position in contact with two discs in two dimensions). It is then rigidly incorporated into the packing. Periodic boundary conditions are considred in the lateral directions. This model is of physical interest since it may correspond to a real experiment made with non-interacting particles which are deposited slowly from a viscous fluid with a very low concentration incoming flux and without shaking. Since when a stable position is reached, the sphere stays permanently in this position, our procedure neglects all many-particle effects such as avalanches. In other words, the deposited sphere has never a sufficient weight to displace the particles on which it sits: this corresponds to the ideal situation of vanishing gravity. 
The two-dimensional random packing of discs made with this algorithm, whose packing fraction is of 0.81 , exhibits very interesting structural properties[5]. If we define as defects the discs having a coordination number different from 4, the density of such defects decreases with height $h$ as $h^{-2}$. Thus, in the assymptotic limit of very large heights, we obtain a defect free random structure. Moreover since in the large height defect free region the unit cells are rhomboedra, this random structure exhibits long range orientational order. The bond orientations are distributed between $30^{\circ}$ and $60^{\circ}$ according to a given characteristic angle distribution function. The resulting structure is very sensitive to perturbations in the initial conditions. If, instead of a basal line, one starts with a line of regularly spaced discs, one generates a periodic structure with well defined bond angles, but if some finite vertical disorder is introduced in this first line, we quickly recover the disordered structure[6].

In three dimensions the structural properties of the random packing of spheres are completely different[7] due to the geometrical frustration between HCP and CFC arrays. The resulting structure, whose packing fraction is 0.5812 , can be considered as a "loose packing" when it is compared with other random packings. The mean coordination number is strictly equal to 6 , but, in contrast with the two-dimensional case, the fractions of spheres having a given number of contacts (ranging from 3 to 10), tend quickly to finite values when the height of the packing is increased. Note that, in both cases, the algorithm can be slightly modified to build heaps: one releases the spheres (or the discs) always along the same vertical trajectory. The heaps built in that way have a angle of repose of about $54^{\circ}$ (in both two and three dimensions) larger than the usual experimental values for sand piles. The difference should be attributed to the neglectance of avalanches.

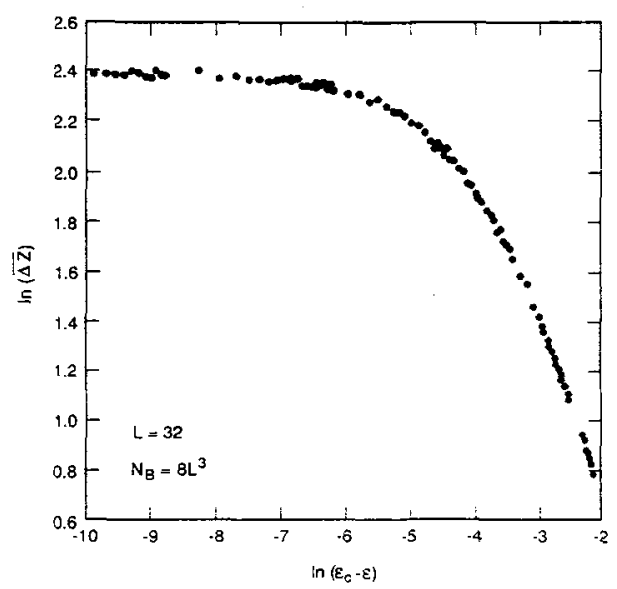

Fig.1.-Mean penetration depth of a small sphere of radius $R_{2}$ in a random packing of large spheres of radius $R_{1}$ as a function of $\epsilon_{c}-\epsilon$, where $\epsilon=\frac{R_{1}-R_{2}}{R_{1}+R_{2}}$ and $\epsilon_{c}=\sqrt{3}-1$.

We have extended the ballistic model in the case of binary mixtures of spheres (or discs) of different diameters. Now, after choosing a random trajectory, we also choose the diameter among two values $R_{1}$ and $R_{2}\left(R_{1}>R_{2}\right)$ with probabilities $X$ and $1-X, X$ being the desired concentration of large spheres. When the size ratio $r=R_{1} / R_{2}$ becomes larger than a critical value, called the Apollonius 
ratio, $r_{c}=\frac{\sqrt{3}}{2-\sqrt{3}}=6.4641 \ldots$ (when a small sphere can penetrate the hole batween three large spheres in contact), a segregation phenomenon occurs and all the small spheres reach the bottom of the packing.

We have systematically studied this segregation phenomenon by calculating the penetration depth of a small sphere into a monodisperse ballistic packing of large spheres[8]. We have built monodisperse ballistic packings of lateral size $L=32 R_{1}$ and we have dropped a small sphere from above the packing along a random vertical and we have calculated the vertical distance $\Delta Z$ separating its stable final position to its position at first contact with the packing. Figure 1 gives the logarithm of $\langle\Delta Z\rangle$, averaged over ten independent packing and, for each packing, over 8000 different verticals, as a function of the logarithm of $\epsilon_{\mathrm{c}}-\epsilon$, where $\epsilon=\frac{R_{1}-R_{2}}{R_{1}+R_{2}}$ and $\epsilon_{\mathrm{c}}=\sqrt{3}-1$. The penetration depth does not diverge as $\epsilon \rightarrow \epsilon_{c}$, but saturates towards a limiting value. This saturation phenomenon is related to the existence, in the large sphere packing, of a finite fraction of equilateral triangles of contacting spheres. Correlatively, near the threshold, the distribution of depths is exponential. This has recently been tested experimentally[9].

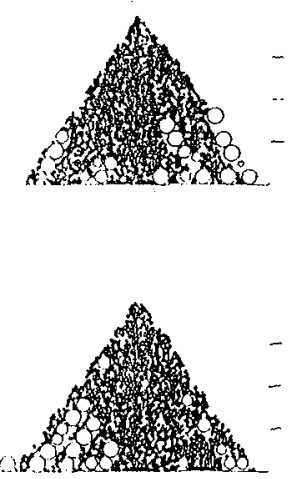

a)

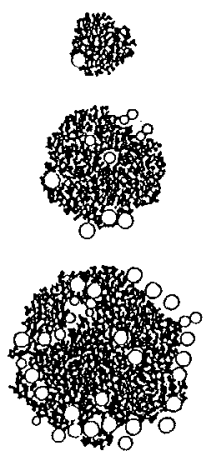

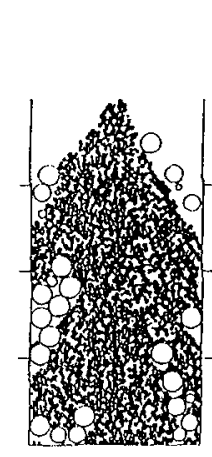

b)

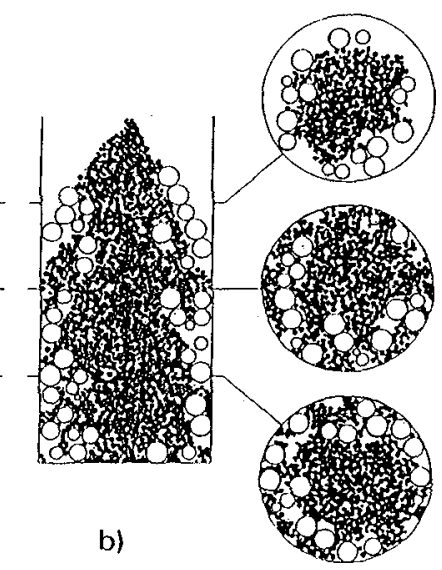

Fig.2.- Vertical and horizontal sections of a packing made with a proportion $X=0.01$ of large spheres and a ratio of diameters $r=4$. (a) Case of a heap. (b) Case of a cylindrical container. The diameter of the cylinder is eight times the big sphere diameter.

Using always steepest descent rules with a binary mixture of spheres but releasing them along the same vertical trajectory, we have simulated the formation of a heap or the filling of a vertical cylindrical container[10]. Here a segregation phenomenon occurs due to the fact that large spheres can more easily roll on a packing of small spheres than the contrary. Figure 2a) shows vertical and horizontal sections of a heap made with a proportion $X=0.01$ of large spheres with a ratio of diameters $r=4$. The characteristic structure of large spheres which are segregated near the periphery of the heap is clearly illustrated. Figure $2 \mathrm{~b}$ ) shows vertical and horizontal sections of a packing made with the same values for $r$ and $X$ in a vertical cylinder with a radius equal to $8 R_{1}$. The big spheres, which accumulate near the wall of the container, disturb the motion of the small ones and are responsible for the formation of large voids. 
We have recently modified the ballistic model to modelize size segregation by shaking[11]. An initial polydisperse packing has been built using the rules of the ballistic model. After the initial packing has been constructed the particles are placed in a list in order of ascending height (of their centers) and then redeposited in that order along the verticals corresponding to their horizontal coordinates and keeping the steepest descent rules. The process of ordering according to height and deposition is repeated many times to simulate the shaking process. Our model corresponds to a large-amplitude and low frequency vertical shaking process in which the duration of a shake is shorter than the time interval between shakes in order that the packing comes completely to rest before the next shake. Very large frictions are also assumed so that spheres do not bounce when landing.

Figure 3 illustrates a typical simulation with $r=4$ where 250 large spheres and 50000 small spheres were initially deposited randomly on a square base of edge $L=16 R_{1}$. Vertical cuts through the deposit are shown for the initial configuration and after thirty and sixty shakes. The upward motion of the large spheres can be clearly seen in this figure.

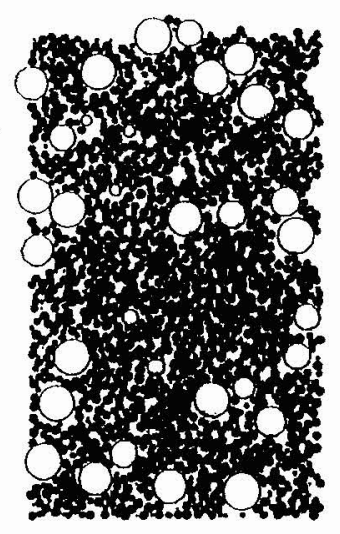

$t=0$

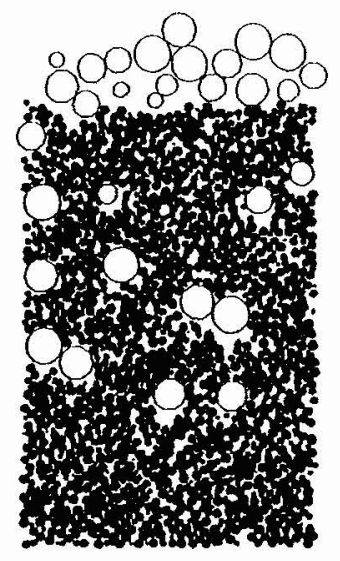

$\mathrm{t}=30$

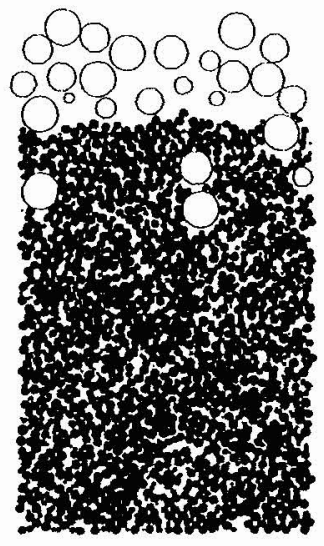

$t=60$

Fig.3.-Vertical sections through a packing with $r=4$ and $X=0.005$. From left to right the initial configurations after thirty shakes and after sixty shakes are shown.

We have observed that the ascending motion of a large sphere becomes non-monotonic and that a large sphere may eventually stop when the size ratio is smaller than a threshold value of about $r_{c}=3$. Both the origin of the rising motion and the existence of a size ratio threshold can be understood by using a simple analytical model that becomes exact in the limit $r \rightarrow \infty$. When a single large sphere imbedded in a packing of small ones reaches a steady state motion of constant velocity, a conical hole lies beneath the large sphere. This cone is characterized by the angle of repose of the small sphere packing. During each shake, the small spheres that are in the anular region (1) of figure 4 will slide into the region (2) before the big sphere comes back. Equating the volumes of these two regions, we obtain:

$$
\frac{\delta}{R_{1}}=\frac{1-\left(1-\cos ^{4} \alpha\right)^{\frac{1}{3}}}{\cos \alpha}
$$


where $\delta$ is the displacement between two shakes and $\alpha$ the angle of repose. For $\alpha=54^{\circ}$ we get $\delta \simeq 0.07 R_{1}$, a value in agreement with the simulations performed with large $r$ values. The threshold occurs when the small spheres are too large for their centers to have a chance to lie in volume (1). Equating the effective volume occupied by a small sphere to the volume of (1), one obtains the estimate:

$$
r_{c}=\left(\frac{4}{f}\right)^{\frac{1}{3}} \frac{\sin ^{\frac{2}{3}} \alpha}{\cos \alpha}
$$

where $f$ is the packing fraction of the small sphere packing. For $f \simeq 0.58$ and $\alpha=54^{\circ}$ we obtain $r_{c} \simeq 3$ as observed in the simulations.

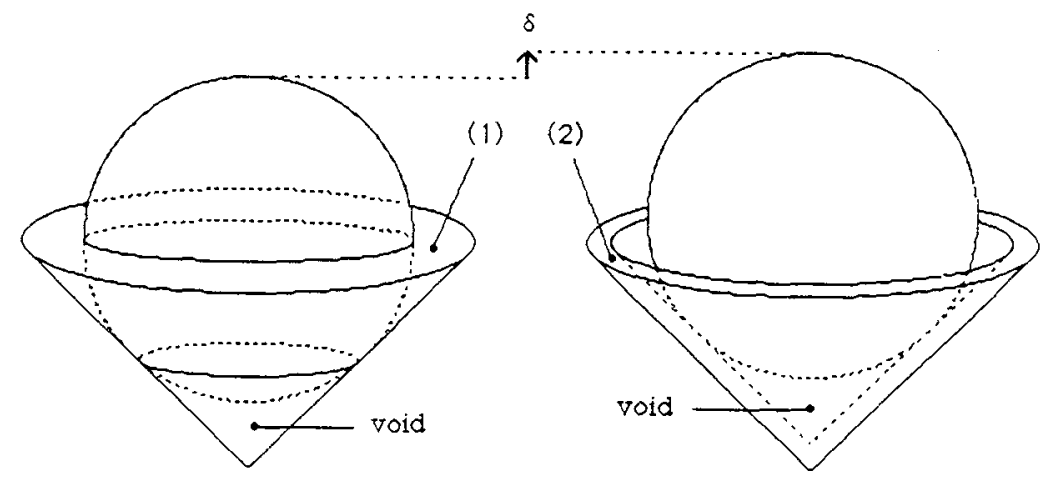

Fig.4.-Sketch of the rising mechanism of the large sphere in the case of an infinite ratio of radii. When a shake occurs, the small spheres which were located in region (1) (below the large sphere center) will come back before the large sphere and will be able to slide along the cavity edges to occupy region (2). The big sphere will then land at a higher position than initially.

In three dimensional experiments, it might be difficult to observe the size ratio threshold. Since the experimental values for $\alpha$ are smaller, $r_{c}$ should be closer to one. The above formula with $\alpha \simeq 30^{\circ}$ and $f \simeq 0.6$ gives $r_{c} \simeq 1.37$. Moreover, if we include some randomness in our model, $r_{c}$ is reduced and the transition is rounded[12]. Similar calculations have been recently done in two dimensions[13] and the size ratio threshold has been found to be considerably larger (of order 12). Such large size ratio threshold has recently been observed in an experiment done with aluminium $\operatorname{discs}[14]$.

In conclusion, models of the type of those described here are very useful since they often lead to very simple physical interpretations of real phenomena. We have reported on two examples for which theoretical predictions were confirmed by experiments: exponential distribution of depths at the Apollonius threshold for the penetration of a small sphere in a random packing of large ones[8-9] and size ratio threshold for segregation by shaking in two dimensions[13-14]. These simple models are complementary of more sophisticated numerical methods like molecular dynamics. 
Acknowledgements.--One of us (R. J.) would like to acknowledge support from the "GDR Matériaux Hétérogènes Complexes" of the CNRS.

\section{References}

[1] Jullien R. and Botet R., "Aggregation and fractal aggregates", World Scientific (Singapore, 1987).

[2] M. J. Vold, J. Coll. Int. Sci., 14, 168, 1959; J. Phys. Chem., 63, 1608, 1959; 64, 1616, 1960.

[3] W. M. Visscher and M. Bosterli, Nature, 239, 504, 1972.

[4] R. Jullien and P. Meakin, Europhys. Letters, 4, 1385, 1987.

[5] P. Meakin and R. Jullien, Europhys. Letters, 14, 667, 1991.

[6] R. Jullien and P. Meakin, J. de Physique (France), 1, 1263, 1991.

[7] A. Pavlovitch, R. Jullien and P. Meakin, Physica A, 176, 206, 1991.

[8] P. Meakin and R. Jullien, J. de Physique, 51, 2673, 1990.

[9] G. Ghidaglia, E. Guazzelli and L. Oger, J. Phys. D, 24, 2111, 1991.

[10] R. Jullien and P. Meakin, Nature, 344, 425, 1990.

[11] R. Jullien, P. Meakin and A. Pavlovitch, Phys. Rev. Letters, 69, 640, 1992.

[12] R. Jullien, P. Meakin and A. Pavlovitch, Phys. Rev. Letters, 70, 2195, 1993.

[13] R. Jullien, P. Meakin and A. Pavlovitch, Europhys. Letters, 22, 523, 1993.

[14] J. Duran, J. Rajchenbach and E. Clément, Phys. Rev. Letters, 70, 2431, 1993. 